injections of cocain for spinal anesthesia found that following the injection of a few centimeters of a cocain solution, patients thus treated suffered from vomiting, fever, headache and more or less neuralgic pain and troubles of urination. At first the origin of these accidents was attributed to the cocain itself or to an idiosyncrasy on the part of the patient. Ravaut and Auberg, ${ }^{5}$ in studying these cases, found that this train of symptoms was actually due to an aseptic, sterile meningitis. They were able to demonstrate clearly, in the spinal fluid, cytologic reactions which proved this contention. They established the fact that a watery solution which was not isotonic could produce meningeal irritation, and by substituting cocain in isotonic solutions for solutions previously used, they were able to show that the patients tolerated this with the desired anesthesia and with perfect impunity to harmful results.

In 1907 Ravaut $^{6}$ showed that hypertonic concentrated solutions were even better tolerated than isotonic solutions. These by reason of their density are not easily diffused. 'Thus he was able to produce complete genital and perineal anesthesia by the injection of a single drop of water containing $2 \mathrm{cg}$. of cocain or novocain. If patients thas treated, however, were placed in the Trendelenburg position, there was a very rapid diffusion of the cocain toward the upper portion of the cord and brain, resulting in nausea and vomiting constantly and within a short time. According to the position of the patient, therefore, it became possible to direct the solution toward the inferior or superior portions of the nervous system.

Ravaut employed the method of concentrated hypertonic solutions in the injection of neosalvarsan in the spinal cord, and he has recently published his results, with an account of patients thus treated. ${ }^{7}$ Using the following method, as outlined to me by Dr. Ravaut, I have been employing this treatemnt at the University Hospital during the past nine months.

The solution used is a 6 per cent. solution of neosalvarsan in distilled water. This solution is hypertonic. Each drop of such a solution would contain $3 \mathrm{mg}$. of neosalvarsan. The dosage injected is from 3 to $12 \mathrm{mg}$.; that is, from 1 to 4 drops of the solution. The solution is made up as follows:

An ampule containing $0.3 \mathrm{gm}$. of neosalvarsan is dissolved in 5 c.c. of freshly distilled water. If the ampule contains $0.6 \mathrm{gm} ., 10$ c.c. of water are used. In both solutions each drop will contain $3 \mathrm{mg}$. of the drug. The syringe employed for the injection is accurately graduated in drops. The patient is then placed in a position for a lumbar puncture, either sitting or lying, according to the choice of the operator. The puncture is then made with a needle, the end of which fits the graduated syringe. After a few drops of spinal fluid have flowed out of the cannula, or a greater quantity if a diagnostic puncture is desired at this time, the syringe is fitted into the needle, and the fluid is allowed to run back into the syringe barrel, thus mixing with the amount of the drug in the barrel. The mixed spinal fluid and drug is then gently forced into the canal, and slight suction is made on the syringe to withdraw a second amount of fluid, which washes out the needle. This is then reintroduced, the needle is quickly withdrawn and the patient placed in the Trendelenburg position, in which position he is allowed to remain for at least one hour.

5. Ravant and Auberg : Compt. rend. Soc. de biol. 1901, liii, 637. 6. Ravaut: Compt. rend. Soc de biol., 1907, Ixii. 1159 .

7. Ravaut: Ann. de méd., 1914, No. 1, p. 49.
Injections thus given are exceefing]y well tolerated. At most there is a slight amount of neuralgic pain, which passes off in a short time. For the most part, however, if carried out properly, the injections are quite painless. A slight amount of neuralgic pain is particularly likely to occur in tabetics. Vomiting does not occur at all. A slight amount of headache such as accompanies a lumbar puncture is, however, sometimes encountered. A slight tendency to difficulty in urination is oceasionally experienced a day or two following the injection. In two cases of my own, it persisted, but in both of these my technic was at fault. I have carried out this treatment in a number of cases, and an account of these will follow in a report to be read before the Section on Dermatology of the American Medical Association in June.

\section{AN UNUSUAL CASE OF VACCINE ANAPHYLAXIS *}

RAYMOND WALLACE, M.D.

Surgeon to Baroness Erlanger Hospital

CHATTANOOGA, TENN.

Anaphylactic reactions following the injection of horse-serum in sensitized individuals are of common occurrence, and the various phenomena in animals sensitized to foreign proteins have been extensively studied. Great effort is now being made to unify these manifestations of anaphylaxis and immunity on a single basis, despite their many striking variations.

'The introduction into the human circulation of foreign proteins. in the form of bacterial vaccines, is usually in such small amount that there is but slight local and usually no appreciable general reaction.

The following case is of special consequence brcause of its rarity in the literature, both of anaphylaxis and of vaccine therapy:

Mrs. S. J. P., aged 53, presented a small furuncle on the left forearm and an infected hair follicle in the right axilla. Three years previously she had suffered from boils and was effectively cured with four serial doses of staphylo-streptobacterin. Similar treatment was requested in this instance, and an injection of fresh stock vaccine, containing 100,000000 streptococci, 400,000,000 staphylococei and 200,000,000 colon bacilli was given. There was only slight local reaction, and considerable improvement resulted in the existing furuncles. On the eighth day at 10 a. $\mathrm{m}$. double the dose of the same vaccine was administered; at $4 \mathrm{p}$. $\mathrm{m}$. there was a diffuse erythema about the lesion on the forearm, and an angry spreading erythema covering the right axilla, upper arm and breast. A nurse was at once placed in charge and records made at three-hour intervals. By 9 p. $\mathrm{m}$. a scarlatinaform erythema had involved the entire trunk and parts of the extremities. There was no sore throat or constitutional disturbance, and the temperature and pulse were normal at all times. On the third day the entire body was involved, the advancing areas were macular, but rapidly became confluent and intensely red, the turgidity of the skin and pruritus was only momentarily relieved by lotions, unguents and dustingpowders, and the suffering was intense. The neck, trunk and parts of the extremities now presented the appearance of a fulminating erysipelas.

On the administration of free catharsis and alkalies, the eruption gradually faded, and on the tenth day there remained but slight erythema and induration about the original lesions. The temperature and pulse at no time varied from normal; there were no throat symptoms, joint pains or other systemic

* Reported before the Clattanooga Academy of Medicine, Feb. 20, 1914 . 
disturbances. Ienkocyte-counts showed but slight numerical or differential changes from normal; the urine contained no albumin, sugar or indican. The erythema, however, was so intense and indurated, that as it disappeared there was considerable fissuring of the skin in the flexures and a mild desquamation in many parts.

Such hypersusceptibility to bacterial proteins as occurred here, is, from the literature, exceedingly rare. With marker anaphylactic reaction, the absence of systemic upset is extraordinary.

The phenomenon itself is undoubted. It oceurred as an intense scarlatinaform erythema, universal in extent and withoat constitutional disturbance. It was not an exanthematous disease, nor was the eruption related as at times, to parenteral protein digestion.

\section{EXOPHTHALAIC GOITER AS A CLINICAL MANIFESTA-} TION OF HEREDITARY SYPHILIS

\section{oscar Clark, M.D., Rio de Janeiro, Brazil}

I have had the opportunity, during the last five years, of observing an interesting case of exoplithalmic goiter in a woman, aged 24, in whom there can be no doubt that it is a late clinical manifestation of hereditary syphilis. The patient began about five years ago to suffer from palpitation tachycardia, accompanied by violent attacks of nervousness, while her mother also called attention to a swelling of the neck. There was very little protrusion of the eyes.

Four years ago when I was first consulted (shortly before leaving on a journey to Europe, during which I was kept informed of the progress of the case), the clinical symptoms had become decisice and the syndrome grew steadily worse until the patient fell into a drowsy, almost comatose condition, with pulse over 200 beats, the sphincters quite paralyzed, with continuous vomiting lasting a fortnight. Dr. Sylvio Moniz, a well-known Brazilian physician, was summoned to the patient in this very bad condition and had the happy thought of trying the Wassermann reaction. To this he was led by the "Olympic forehead" and the crûne natiforme, being told also that the girl when 12 years old, had had several "epileptiform" fits. The Wassermann reaction was strongly positive, both with the patient's mother and with the patient herself.

With this diagnosis, Dr. Sylvio Moniz prescribed mercurial frictions and epinephrin (1:1,000 solution, 20 drops three times daily ) to relieve the arterial hypertension and the vomiting; the latter rapidly ceased, while the patient had in two days recovered consciousness.

After eight days the pulse was just 150 and the goiter sensibly smaller, while the protrusion of the eyes was a little better. After thirty frietions the patient was much better both as regards the hyperthyroidic manifestation and the general health. The next month she was given two injections of salvarsan $(0.3$ and $0.6 \mathrm{gm}$.). By the third month she had quite recovered. Neither the goiter nor the protrusion of the eyes was to be seen. The pulse was 75 beats and the nerv ousness had disappeared.

The patient returned to North Brazil (she was at Rio de Janeiro), and ceased the mercurial and salvarsan treatment. Twelve months later, however, the Merseburger triad reappeared but a fresh recovery has been effected by the same treatnent.

I think that this case is undoubtedly one of hereditary syphilis appearing late, as Dr. Sylvio Moniz made every laboratory test in order to exclude tuberculosis, malaria, etc. The father is dead, of what cause I am not aware; the positive Wassermann in the mother has been mentioned. The patient is from the best society and is virgin and there can be no suspicion of syphilis by contagion. She has never had any clinical sign of infection, but on the other hand, presents skeletal stigmata, and, as previously remarked, had epileptiform fits at the age of 12 .

\section{A CASE OF DISSEMINATED CARCINOMA OF THE BREASTS AND AXILLAE IMPROVED BY RADIUM *}

Sixclatr Tousey, M.D., New York

The patient, a man, was referred to me by Dr. Beder. The right manmary gland and nipple had ulcerated away and there was another ulcer below that region. Both were adherent and covered with a red parchment-like epidermis and were surrounded by an indurated margin projecting $1 / 2$ inch above the level of the skin. Nodular masses larger than a hickorynut but smaller than a hen's egg were present in both axillae and were widely scattered over the front of the chest. On the right side of the chest the skin over many of these was red, and they were evidently in a way to break down into ulcers. On the left side the nodules were smaller, white, and extended from the nipple in a cord-like mass up into -the axilla. The disease had been of several years' duration; no operation even for the removal of a microscopic specimen had been permitted. There was no history of syphilis, and two careful Wassermann examinations were negative. The clinical diagnosis had always been carcinoma, and if so it was evidently similar to the cases of epithelioma cicatrisans, which are sometimes active for as long as eight years before causing death. My patient had lost weight and strength, the skin was adherent to the chest wall, and the use of the right arm was interfered with.

Treatment was by contact applications of a mass of chemically pure radium sulphate containing $20 \mathrm{mg}$. of radium element having an activity of 20,000 microcuries, in a sealed glass tube $0.3 \mathrm{~mm}$. in thickness, enclosed in aluminum $0.5 \mathrm{~mm}$. thick and in thin, soft rubber. Treatments were three times a week for a month and after that once a week. At each treatment several individual nodules or parts of the nodular borders of the ulcers received an application equal to a total of fifteen minutes in each place. During the first month every growth on the right side had been treated in this way and also the floor of the ulcers, but the left side had not been treated. At the end of this time all the nodular masses on the right side were perfectly flat, and the cicatrized ulcers were no longer adherent but could be raised and bent double. The arm could be raised to the greatest extent. The patient felt stronger and had gained 5 pounds in weight. The left side, untreated, showed marked improvement, strengthening my belief that the application of the Roentgen ray and radium rays to cancer develops some antibody which is carried through the system and in proper dosage produces benefit to cancer foci beyond the effective reach of the direct rays themselves.

Treatment was then begun on the left side, and the subsequent course of the case has been one of steady progress.

850 Seventh Avenue.

The Favorite Formula or Pet Prescription.-Every physician, whether optimistic or pessimistic in his general attitude toward the materia medica, usually has a favorite formulaa certain combination of drugs which he uses for many and varied selected conditions. It is his favorite formula; here is mine:
P. Codein sulphat Acetphenctidin Aspirin
(15)
Make eight capsules. Une every hour or two.
gr. ii gr. $x x x i$

With this formula, under appropriate conditions, the cure of patients becomes strictly a case of cito, tuto et jucunde, as we are admonished it should be. It will symptomatically cure grip, and if given on the first day will in many cases prcduce such an improvement by the second as to prevent its complications and incidentally-malum in se-do away with the doctor. I have used the favorite formula with satisfaction in most inflammations. There are other indications naturally suggested by its ingredients to which it seems excellently adapted and which it is unnecessary to enumerate.-Wilfned M. Bartox, M.D., Washington, D. C.

* Case shown at the Surgical Section, New York Academy of Medicine, March 6, 1914. 